\title{
On Some Future Developments in Polymer Science
}

Text by Stanislaw PENCZEK

As it is well known, no future can be predicted with a high enough certainty. We witnessed in the past statements concerning the future of polymer chemistry that have never been materialized. For instance, at the early 1950-ies one of the leading scientists stated, that as far as the large scale commodities, are, the polymer chemistry and technology exhausted its possibilities. And then, just almost overnight a few years later the Ziegler-Natta discoveries came. Moreover, in the post-war time (late 1940s) in several countries (France, Japan) there were high hopes on the radiation polymerization. In spite, however, of the sophisticated and large scale research conducted in a number of countries, there is not too much left (except irradiated PE) in the practical use. The same could be said about the polymer drugs (DIVEMA is the striking example). Therefore predicting the future, as required for these pages is almost impossible.

There are also today scientists saying that there is no future for any large scale polymer in future. The ample evidence for these views was a recent discussion at the Rolduc Meeting (2006) in the Netherlands. I am not in agreement with this view point. Mostly, because I am convinced, that whatever is thermodynamically allowed and, e.g. realized in Nature, will eventually by the industrial reality. I particularly have in mind bio-synthesis and/ or genetically modified natural polymers, i.e. cellulose. If I were younger I would certainly choose working in such an area, hoping that slightly modified cellulose, with reduced number of H-bonds, could provide tractable material when the crystallinity would be sufficiently decreased, allowing melting before decomposition comes. Development of enzymes for efficient hydrolysis of cellulose to simple sugars could be another important future development. There are good examples for such approaches in the already conducted research, namely in Prof. D. Tirrell's biosynthesis of polypeptides and the work in Japan, conducted by the group of Prof. Y. Doi, showing that one could get various bacterial polyesters by modifying the responsible for their structures parent structures. Other future developments might belong to superstructures (dynamers) developed by Prof. J. M. Lehn. Polymer technology requires that thermoplastics are melted and then shaped by extrusion, injection molding and related methods. These are energy consuming processes. One could dream, that further development in the dynamers would bring a possibility of mixing two liquids comprising components giving instantaneously a solid polymer of desired properties, like already realized today mixing of two homochiral polymers giving stereocomplexes of superior properties.

Something has to happen to the chemical literature: to this huge avalanche of papers that provide information that one single person could not absorb anymore, even in the relatively narrow fields. Commercial journals became expensive (e.g. subscription of one of the journal in the field of polymers costs, for instance, \$11 K USD/year). There are increasing numbers of journals, since it is quite profitable business. Large publishing companies are

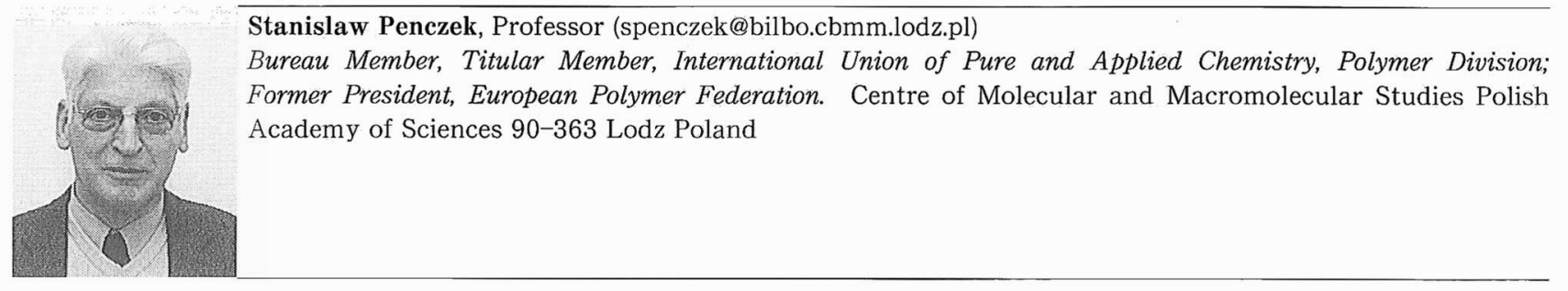


fighting for the market share and, in general, as I do observe it over the years, the average quality of accepted papers decreases. As a referee to several journals I could also say, that it happens that apparently due to the idea "the more-the better" Editors are accepting papers having even two negative opinions. Taking into account an increasing number of the very good papers coming from China and India, the much more scrupulous approach of the Referees and Editors should be taken in order to eliminate the poorer ones. The Open Access Initiative (OAI) may change this lamentable avalanche of the very poor papers and Japan, and particularly its Society for Promotion of Science as well as Society for Polymers in our field could play in this area a particularly important role.

For many years I have had a pleasure and privilege to be associated with eminent Japanese Scientists. I have also spent by their invitation almost two months some years ago, visiting, in the carefully planned tour, 11 towns and over 25 Universities (from Sendai to Fukuoka). This tour allowed me to complement my understanding of the polymer science and there is no doubt that influenced the research my own. 